\section{PWE-018 EVALUATION OF THE BOWEL SCREENING WALES NETWORK MULTIDISCIPLINARY TEAM AND NATIONAL REFERRAL CENTRE PILOT}

doi:10.1136/gutjnl-2013-304907.307

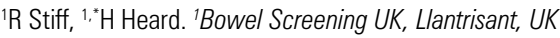

Introduction The Bowel Screening Wales (BSW) Network Multi Disciplinary Team (NMDT) and National Referral Centre (NRC) pilot was established in October 2011 to offer the opportunity for expert opinion and discussion of therapeutic options for participants with complex benign polyps detected on the Welsh bowel screening programme. Formal evaluation of the pilot was undertaken to inform future service development and focused on establishing the views of participants.

Methods All screening participants with complex benign polyps referred to the NMDT between the $1^{\text {st }}$ of January and the $17^{\text {th }}$ of April 2012 were included in the evaluation.

Telephone interviews were conducted by members of BSW staff without direct involvement in their care using a structured questionnaire. Questions were of two different formats: some required responders to choose from a selection of structured options; whilst others required free-flowing text responses. Both qualitative and quantitative analysis methods were used.

Results A total of 41 screening participants were referred to the NMDT during this time frame. Twenty four participants agreed to take part in the evaluation (59\%). Participants who responded were geographically dispersed and had a variety of management outcomes following index screening colonoscopy.

The importance participants placed on being involved in decision making about their care was emphasised by nineteen (79\%) responders who considered it essential or important that a decision by the local team to refer their case to the NMDT was discussed with them prior to referral.

Most responders (92\%) reported that following index colonoscopy, management options were discussed with them. Twenty one responders $(87 \%)$ reported that they had received sufficient information for them to be able to make an informed choice about the best clinical option for them.

Although overall awareness of the NMDT and NRC pilot among screening participants was low (only 5 responders- $21 \%$ were aware), nine participants (38\%) reported they were aware that their local team could seek advice about their clinical management from a group of experts outside of the immediate local team.

Participants 'opinion about the overall service they received from the NRC was favourable, with $6(86 \%)$ individuals describing the service as excellent and one individual describing the service as good.

Conclusion The BSW NMDT and NRC pilot was established to facilitate informed choice and to develop an equitable service for participants of the bowel screening programme in Wales. Evaluation of the initial pilot stage confirmed that participants felt involved in decision making about their treatment options and that the service was received positively. Initial evaluation would suggest that the pilot met the aim of developing an equitable participant focused service.

Disclosure of Interest None Declared.

\section{PWE-019 PATIENT EXPERIENCES OF A POLYP CANCER IN THE BOWEL CANCER SCREENING PROGRAMME}

doi:10.1136/gutjnl-2013-304907.308

1.2, H Miller, '1 Sedgewick. 'School of Health and Social Care, Teesside University, Middlesbrough; ' ${ }^{2}$ South of Tyne Bowel Cancer Screening Centre, Gateshead Health NHS Trust, Gateshead, UK

Introduction This study explores the experiences of patients in relation to their treatment modality to examine if there are any differences between patients who had endoscopic treatment vs. surgical treatment of a polyp carcinoma.

Methods 49 patients diagnosed with a polyp cancer through the NHS Bowel Cancer Screening Programme (NHSBCSP). 24 patients (endoscopic management) and 25 patients (surgical management). A mixed-method, two-phase explanatory sequential design was employed. Phase One used mailed, EO5D5L quality of life questionnaires to identify any general quality of life issues between surgical and endoscopic groups. Phase Two comprised of face-to-face, in-depth interviews to explore the patient experience in more detail.

Results A difference in QoL issues surrounding self-care was identified during phase $1(p<0.016)$. Phase 2 highlighted that patients had little or no awareness of the NHSBCSP prior to participation. Most patients were provided with treatment options and felt involved in the decision-making process. Those managed surgically $(n=3)$ had regular contact and support from surgical and colorectal nursing teams whilst endoscopic patients $(\mathrm{n}=2)$ only had a single point of contact. All patients $(n=5)$ experienced feelings of shock, disbelief and fear following diagnosis with particular focus upon fear of a colostomy (surgical patients $n=3$ ) and fear of cancer recurrence $(\mathrm{n}=5$ ).

Conclusion Patients diagnosed with a polyp carcinoma experience the same fears and anxieties despite treatment modality however, follow-up care and support differs significantly for the two groups with surgically managed patients being provided with more professional, clinical support than their endoscopically managed counterparts.

Disclosure of Interest None Declared.

\section{PWE-020 DO SEASONAL TRENDS IN POSITIVITY EXIST WITH THE GUAIAC-BASED FAECAL OCCULT BLOOD TEST?}

doi:10.1136/gutjnl-2013-304907.309

1,2, H E Seaman, 'P Butler, '1 M M Snowball, 1,2S P Halloran. 'NHS Bowel Cancer Screening Programme, Southern Hub; 'University of Surrey, Guildford, UK

Introduction Several bowel cancer screening programmes, including the NHS Bowel Cancer Screening Programme (BCSP) in England, use a guaiac-based faecal occult blood test (gFOBt). Seasonal variations in gFOBt positivity have been reported, with lower positivity in the warmer spring and summer months compared with autumn and winter. As more population-based bowel cancer screening programmes are being planned and implemented worldwide, it is important to highlight the factors that could affect the performance of faecal occult blood tests.

Methods Men and women aged 60-69 years are eligible for biennial routine bowel cancer screening by the BCSP. The gFOBt card comprises six windows (or 'spots') onto which the screening participant is asked to apply two samples from three separate stools. Test cards are returned to one of five BCSP Hubs where each spot is tested to determine 'spot positivity'. The number of positive spots determines test positivity: $5-6$ positive spots = positive test and immediate referral for colonoscopy; $1-4$ positive spots $=$ weak positive and re-test. Ultimately, a definitive test result is achieved for each screening episode, according to a well-defined test/re-test algorithm. Data from the Bowel Cancer Screening System (BCSS) for the BCSP Southern Hub for April 2008 to March 2012 were analysed and gFOBt spot positivity patterns assessed by month, stratified by age and sex. Analysis was limited to the first (prevalent) screening episode for all individuals.

Results In total, 7,765,282 spots were analysed (nearly 1.3 million kits). As expected, throughout the study period, gFOBt spot positivity for men $(2.6 \%)$ was consistently higher than for women $(1.7 \%)$ and increased with age. Any variations in seasonal positivity were similar for men and women and all ages. The pattern of spot positivity by month was not consistent year on year and there was 